\title{
Aprendizaje Basado en Retos con DT en Temas de Lingüística General, optativa de cuarto del Grado en Filología Hispánica
}

\section{Challenge Based Learning with DT in Topics in General Linguistics, a fourth-year elective of the Degree in Hispanic Philology}

JuAn Pablo Mora GutiÉRREZ

ORCID https://orcid.org/0000-0002-8614-5117

Universidad de Sevilla

Área de Lingüística General

Departamento de Lengua Española,

Lingüistica y Teoría de la Literatura

jmora@us.es

Fecha de recepción: 12-11-2019

Fecha de aceptación: 17-11-2019

DOI: http://dx.doi.org/10.12795/9788447221912.060 Pp.: 1374-1401 


\section{Resumen}

Este artículo trata sobre un Ciclo de Mejora en el Aula (CIMA) llevado a cabo durante el curso 2017/2018 en la asignatura optativa de seis créditos de cuarto de Filología Hispánica de la Universidad de Sevilla (US) Temas de Lingüística General. La innovación se centró en el uso del modelo docente Aprendizaje Basado en Retos (ABR) con metodología de Pensamiento de Diseño (Design Thinking, DT) adaptada como ECO en el marco de un proyecto de innovación docente en la US cuyo principal objetivo fue el trabajo en equipo de profesorado y alumnado para hacer frente a retos de la sociedad. Esta metodología constructivista fue acompañada por una evaluación continua a través de portafolios grupales y de un proceso de gamificación. Los resultados fueron mucho mejores que los conseguidos el curso 2016/2017 en el que se usó ABR pero sin metodología DT/ECO ni gamificación.

Palabras clave: Lingüística, Grado de Filología Hispánica, docencia universitaria, experimentación docente universitaria, Aprendizaje Basado en Retos.

\section{Abstract}

This article deals with a Cycle of Improvement in the Classroom (CIMA) in a 6 credit fourth-year course Topics in General Linguistics of the Degree in Hispanic Philology of the University of Sevilla during the 2017/2018 academic year. The CIMA revolved around the use of Challenge Based Learning with Design Thinking adapted to ECO in a teaching innovation project at US whose main objective was the work in teams of teachers and students to face needs and challenges of society (CBL with DT/ECO). The continuous evaluation was carried out through portfolios and gamification. The results were much better than the ones obtained in 2016/2017 in which Challenge Based Learning was used but without DT/ECO and without gamification.

Keywords: Linguistics, Hispanic Philology, Higher Education, University Teaching Experimentation, Challenge Based Learning.

Jornadas de Formación e Innovación Docente del Profesorado | № 2 (2019) Esta obra se distribuye con la licencia Creative Commons 


\section{Breve descripción del contexto de la intervención}

Este trabajo cuenta la experiencia de innovación docente que llevé a cabo durante el segundo cuatrimestre del curso 2017/2018 en la asignatura optativa de 6 créditos de cuarto curso del Grado en Filología Hispánica Temas de Lingüistica General en el marco de un proyecto de innovación docente del Tercer Plan Propio de Docencia de la Universidad de Sevilla Aprendizaje como proceso de diseño: innovación universitaria desde el Design Thinking. En este proyecto participó profesorado (11) y alumnado (más de 600) de varios Grados (Bellas Artes, Restauración y Conservación de Bienes Culturales, Comunicación Audiovisual, Filología Hispánica, Estudios Ingleses, Edificación y Pedagogía) y Másteres (MAES, MASELE, MUSIE y FOT). Durante el curso docente 2018/2019 este proyecto docente continuó con 22 docentes y más de 1350 estudiantes de 5 universidades con el título ECO en la Educación Superior. Docencia inspirada en el entorno y en el curso 2019/2020 somos casi cuarenta los docentes comprometidos para usar ECO en nuestra docencia.

El objetivo fundamental del proyecto de innovación docente fue propiciar el trabajo conjunto de profesorado y alumnado de materias diversas a través de un modelo docente, el Aprendizaje Basado en Retos (ABR) con metodología de Pensamiento de Diseño o DT (Design Thinking), que cambia el enfoque de la enseñanza al aprendizaje. No se persigue ni el protagonismo del profesorado ni el del estudiantado sino que se pone el foco fuera del aula, en los problemas o retos a los que se enfrenta la sociedad. El alumnado tiene que encontrar y definir un reto o problema, empatizar con las personas a quienes concierne ese reto y explorar las soluciones que se hubieran implementado anteriormente para crear e implementar una nueva solución viable, innovadora y creativa. Por último, durante las diversas fases del DT es necesario presentar 
las propuestas a las personas afectadas para modificarlas cuantas veces fuera preciso para adaptarlas a las necesidades reales de la sociedad y de esas personas.

De los seis créditos de la asignatura me correspondían a mí cuatro oficialmente. Los otros dos los impartía Diego Jiménez. Acordamos que él se encargaría de implementar una dinámica de gamificación (Jiménez, 2017) por la que el alumnado empezaría con una nota inicial de 10 siguiendo lo expuesto en Chou (2016) y lo recomendado en el Eabe 2017 de Almería por José Luis Castillo (@jlcastilloch). A esta nota inicial se le podría sumar puntos a través de una serie de retos e incentivos, o restar, si el trabajo que se iba desarrollando no era de 10. Diego Jiménez también usó esta experiencia como su CIMA del Curso General de Docencia Universitaria del Programa de Formación e Innovación Docente del Profesorado de la US (Jiménez, 2018).

En la asignatura se matricularon oficialmente 57 alumnas y alumnos, 36 estudiantes del Grado de Filología Hispánica y los 21 restantes eran alumnado Erasmus procedente de diversos países europeos. Con las 47 personas que optaron por la asistencia regular a clase y el modelo ABR con DT se formaron 10 equipos con la única restricción de que, si era factible, en cada equipo tenía que haber obligatoriamente alumnado español y extranjero para favorecer el intercambio intercultural y la diversidad de formas de trabajo. Dos alumnas y un alumno optaron por llevar a cabo proyectos individuales, la primera por no poder asistir a clase por enfermedad, la segunda por encontrarse en el extranjero y el tercero por motivos laborales. Diecisiete habían tomado conmigo en el curso 2014/2015 o en cursos anteriores la asignatura troncal de primero Lingüística en la que habíamos usado el modelo docente de Aula Invertida, Flipped Learning (Bergmann y Sams 2012), complementado con Aprendizaje Basado en Proyectos y Aprendizaje Cooperativo (Mora 2015, 2017a y 2017b). Además, trece alumnos habían cursado durante el primer 
cuatrimestre del curso 2017/2018 conmigo o en el grupo de la tarde de la profesora Victoria Camacho, la asignatura Lingüística Aplicada, optativa de tercero de Filología Hispánica en la que habíamos usado por primera vez ABR con DT con la ayuda de Eduardo Herrero, de ETSIE, uno de los docentes promotores del proyecto de innovación docente. También fue muy relevante la experiencia vivida el curso 2016/2017 en esta misma asignatura Temas de Lingüística General y que fue objeto del CIMA de la REFID contado en Mora (2017b) y que se basó en el empleo del modelo ABR pero sin DT ni gamificación. El curso 2016/2017 fue el primero en el que implementé ABR en mi docencia.

El aula en la que se desarrolló esta innovación docente es una de las clases más grandes de Filología, el aula 203 "Manuel Ariza". Aunque por la disposición del mobiliario invita a una docencia tradicional, centrada en el docente y en los contenidos, era lo suficientemente amplia como para que 10 equipos pudieran trabajar simultáneamente, aunque no cómodamente por tratarse de bancas de madera con poca superficie de trabajo.

\section{Diseño del CIMA}

\section{Contenidos y problemas o preguntas-clave}

En un modelo docente basado en la enseñanza y centrado en el docente y en los contenidos, la asignatura Temas de Lingüística General se presta a que quien la imparta pueda elegir el contenido de la materia que más le convenga por sus intereses docentes o investigadores, ya que ni en el título ni en el programa se preestablece ningún contenido concreto. En un modelo docente constructivista de ABR con metodología DT como el que se decidió implementar en el curso 2017/2018 es el propio alumnado 
el que elige en equipo los temas que va a estudiar y en los que se va a centrar. Así pues, todo el contenido se construye al mismo tiempo que se desarrolla la asignatura y depende enteramente de los intereses del alumnado y de las necesidades y retos de la sociedad que el alumnado decide acometer.

Paso a describir a continuación los retos que diseñaron y a los que se enfrentaron los diez equipos de la asignatura. Ponen de manifiesto la diversidad de contenidos lingüísticos y no lingüísticos que se han abordado y construido durante el cuatrimestre y la diversidad de voces e historias que se generaron en la asignatura:

Equipo 1: Quítate la máscara sobre humor andaluz y su enseñanza al alumnado Erasmus. Este equipo lo constituían tres alumnas españolas, una austríaca y una alemana.

Equipo 2: Con la venia de su senoría: Quid iuris sobre la complejidad del lenguaje jurídico y cómo lograr que las nuevas generaciones de juristas lo modernicen y simplifiquen. Este equipo estaba formado por cuatro alumnas españolas y una alemana: https: / / forense.blogspot.com/

Equipo 3: La Libertad también se escribe sobre enseñanza de español académico a antiguos guerrilleros de las FARC de Colombia, equipo formado por tres españoles, una colombiana y un alemán.

Equipo 4: La Unión hace la Fuerza sobre la enseñanza de lengua y literatura a alumnado Asperger de secundaria y universitario, formado por tres españolas, una alemana y una checa: https://asperger-us.jimdofree.com/

Equipo 5: ALAS sobre enseñanza de gramática del español a alumnado Erasmus, formado por un español, un austríaco, un alemán, una italiana y una polaca.

Equipo 6: Heroes Academy sobre enseñanza de lengua a alumnado de primaria y secundaria convaleciente de cáncer. Este equipo estuvo formado por tres españolas 
y una alemana: https://masquesupervivientes.blogspot. $\mathrm{com} /$

Equipo 7: Portavoces de lo Inefable, que se planteó el reto de enseñar español como segunda lengua de una forma más amena y efectiva a alumnado refugiado. Este equipo lo formaron dos alumnas españolas, una alumna belga y un alumno eslovaco: https://portavocesdlinefable. wixsite.com/portavoces-inefable

Equipo 8: Gamificación: la solución es jugar... o no, con el reto de gamificar la enseñanza de lengua y literatura de un curso de la ESO, formado por una española, dos francesas y un italiano.

Equipo 9: La Forma del Aula trabajó sobre el reto que supone la educación del alumnado sordo de secundaria y el papel que debería desempeñar la LSE, formado por cuatro españoles y una alemana https://formadelaula.jimdo. $\mathrm{com} /$

Equipo 10: Los alemanes tienen un animal en la cabeza, sobre palabras intraducibles del alemán y cómo enseñarlas al alumnado español que aprende alemán, formado por tres españoles y dos alemanas https:/ /grupo10linguistica.wixsite.com/misitio

Los tres alumnos que trabajaron por su cuenta por no poder asistir a clase desarrollaron un proyecto sobre enseñanza de ELE a migrantes de origen chino, de ELE a alumnado de secundaria francés y de enseñanza de la Fonética y Fonología a alumnado de Filología.

En esta entrada de mi blog se puede acceder a la mayoría de los productos de los retos: https://jpmoragu. wordpress.com/2018/07/01/design-thinking-haciendonos-eco-en-las-clases-de-linguistica-aplicada-y-temas-de-linguistica-general/. En este padlet se puede acceder a proyectos desarrollados por mi alumnado en esta y otras asignaturas impartidas por mí entre 2016 y 2019: https://padlet.com/jmora4/ax4pbgolrwfo 
El principal objetivo de esta experiencia de innovación docente fue intentar conseguir que el alumnado viera la relevancia de la lingüística y de los lingüistas en la solución de muchos de los retos y problemas que tiene la sociedad. Otro objetivo consistió en que los futuros profesionales a los que formo pudieran aplicar lo aprendido en la carrera a necesidades reales sobre las que los lingüistas y la lingüistica tienen mucho que aportar. No menos importante fue que el alumnado desarrollara competencias transversales como el trabajo en equipo o ser capaces de presentar los logros y resultados a través de diversos formatos como son el póster, el vídeo, el portafolios, los blogs o la comunicación oral y escrita.

Por último, ya que la mayoría de mi alumnado se va a dedicar profesionalmente a la docencia, quería que vivieran y protagonizaran una experiencia docente en la que se pusiera de manifiesto que metodologías docentes alternativas a la tradicional centrada en el docente y los contenidos son posibles y deseables para que les sirviera de modelo en su futuro como docentes. De esta forma aspiro a un efecto multiplicador de lo que hago.

\section{Modelo metodológico y Secuencia de Actividades}

Para conseguir estos objetivos, el modelo docente empleado fue el ABR con metodología DT. El Pensamiento de Diseño es una metodología que se usa para conseguir resultados novedosos y creativos para todo tipo de problemas o retos de un colectivo en cualquier ámbito. Su uso en educación está creciendo en los últimos años (Mcintosh 2014). En esta metodología se siguen cinco etapas: Empatiza, que sirve para conocer el problema o reto y empatizar con las personas que se van a ver beneficiadas, para aprender qué se sabe del tema y qué soluciones se han dado hasta la fecha; Define, que sirve para concretar el reto al que se enfrentará el alumnado; Idea, que sirve para imaginar muchas posibles soluciones que se podrian buscar entre las que hay que elegir la más efectiva, innovadora y creativa, pero que a su vez sea viable; Prototipa, 
fase en la que se construye un modelo o prototipo de la idea seleccionada y se presenta a la sociedad y a las personas para las que se trabaja para comprobar si se adapta a sus necesidades y para recibir feedback; por último, Evalúa, para implementar y evaluar la solución a la que se ha llegado. Nosotros hemos añadido una sexta etapa muy pertinente para nuestra labor docente que es la de Ofrecer o presentar el resultado final a la sociedad. Por otra parte, también en el marco del proyecto de innovación docente hemos creado una adaptación de la metodología DT a nuestro contexto universitario español. A esta adaptación la hemos denominado método ECO pues intentamos hacernos ECO de las necesidades de nuestro entorno. El método ECO tiene tres fases: Explorar, Crear y Ofrecer. En la tabla que sigue, creada por Eduardo Herrero, se ponen en relación las fases del DT con las del método ECO y se enumeran los verbos de las actividades que el alumnado tiene que realizar en cada una de ellas para implementar con éxito la solución al reto creado y diseñado por el propio alumnado:

Tabla 1. Etapas y pasos del método ECO, inspirado en el Pensamiento de Diseño o Design Thinking

\begin{tabular}{|c|c|c|}
\hline \multicolumn{2}{|c|}{ ETAPAS } & PASOS \\
\hline Método ECO & Método DT & \\
\hline \multirow{2}{*}{ EXPLORAR } & EMPATIZA & $\begin{array}{l}\text { Observar } \\
\text { Comprender } \\
\text { Descubrir }\end{array}$ \\
\hline & DEFINE & $\begin{array}{l}\text { Procesar } \\
\text { Elegir un punto de vista } \\
\text { Formular el reto }\end{array}$ \\
\hline \multirow{3}{*}{ CREAR } & IDEA & $\begin{array}{l}\text { Imaginar } \\
\text { Establecer criterios de selección } \\
\text { Elegir entre alternativas } \\
\text { Decidir idea/solución }\end{array}$ \\
\hline & PROTOTIPA & $\begin{array}{l}\text { Construir } \\
\text { Someter a interacción } \\
\text { Reconstruir }\end{array}$ \\
\hline & EVALÚA & $\begin{array}{l}\text { Ganar comprensión } \\
\text { Aplicar retroalimentación } \\
\text { Refinar solución }\end{array}$ \\
\hline OFRECER & COMPARTE & $\begin{array}{l}\text { Implementar } \\
\text { Difundir } \\
\text { Contagiar } \\
\text { Celebrar }\end{array}$ \\
\hline
\end{tabular}

Jornadas de Formación e Innovación Docente del Profesorado | № 2 (2019) Esta obra se distribuye con la licencia Creative Commons Reconocimiento-NoComercial-SinObraDerivada Internacional (CC BY-NC-ND 4.0.) 
Uno de los elementos centrales del método ECO (igual que del DT) es el aprendizaje cooperativo para que se produzca aprendizaje entre pares y se desarrolle una de las competencias clave que demanda la sociedad actual, el trabajo en equipo. Es por ello por lo que se decidió tras consultar al alumnado que la mayoría del tiempo de clase se dedicaría al trabajo en equipo en Proyectos de Inicio de Carrera (PIC) que se orientarían a resolver necesidades y retos que respondieran a problemas reales de la sociedad. Asimismo, se decidió que se llevarían a cabo dos presentaciones formales en clase, a mitad del cuatrimestre y al final, así como otras presentaciones en algunas de las fases de DT, como la fase de prototipado. Por otro lado, se animó al alumnado a participar en diversos eventos relacionados con la divulgación científica como la Feria de la Historia, la MUAC, la Feria de la Ciencia o la Noche de los Investigadores. Por otra parte, se puso como requisito presentar los diversos prototipos a las personas destinatarias de la intervención e incluso en las asociaciones o instituciones con las que colaborara el alumnado. En el marco del proyecto de innovación docente se decidió que todo el alumnado presentaría y ofrecería a la sociedad los resultados de los proyectos en un showroom al final de cada cuatrimestre \#ECOshowUS. También se estableció que, para presentar en otros formatos transmedia y de manera creativa los hitos y logros de sus PIC, los equipos crearían un portafolios, un vídeo de corta duración y un póster. Por último, se decidió que se consultaría al alumnado y se le pediría su opinión sobre cada una de las actividades que se desarrollarían en clase y sobre todo lo relacionado con la evaluación y la calificación gamificada.

\section{Evaluación del estado inicial y final del alumnado}

Puesto que llevo ya varios años impartiendo las asignaturas Lingüística (obligatoria de primero), y las optativas Lingüística Aplicada y Temas al alumnado del grado de 
Filología Hispánica y además pertenezco a la Comisión de Garantía de este grado, sé que el estudiantado crece, madura y aprende mucho durante la carrera. Este crecimiento se produce gracias sobre todo a las lecturas de obras literarias y científicas, a la formación que reciben en las asignaturas tanto de lingüística y lengua española como de literatura, a las estancias Erasmus en el extranjero y a las diversas actividades que se desarrollan en la facultad.

También sé que no tienen demasiadas oportunidades de aplicar los conocimientos aprendidos a la realidad, ni de realizar presentaciones orales o de trabajar en equipo, ni muchas veces de tener su propia voz o de desarrollar pensamiento crítico complejo, al impartirse la mayor parte de la docencia del grado a través de modelos docentes basados más en la enseñanza que en el aprendizaje, con protagonismo del docente y centrados en los contenidos de las materias. A pesar de ello, se empiezan a observar cambios importantes en los últimos años, en parte gracias a la incorporación en los cursos de formación del FIDOP de la US y en la REFID de docentes de la facultad (Bravo 2016 y 2017, Mancera, 2016, Camacho 2019). La razón por la que me animé el curso pasado a usar en Temas un modelo de aprendizaje cooperativo como el ABR y este año a innovar introduciendo la metodología DT/ECO ha sido precisamente para reforzar estas competencias que les van a ser esenciales para poder desenvolverse en el ámbito profesional.

Por último, se decidió que la evaluación de la evolución y aprendizaje del alumnado se llevaría a cabo a través de la observación permanente en clase, de las presentaciones, de los productos de los proyectos, que incluirían los prototipos desarrollados, el póster, el vídeo y sobre todo el portafolios grupal en el que se tendría que describir con detalle lo conseguido y profundizado en el PIC. El portafolios final se tendría que presentar en una breve entrevista oral con los docentes el 20 de junio de 2018 a la hora estipulada para el examen oficial de la asignatura. 
El portafolios final tenía que incluir un diario de clase reflexivo, así como una sección de autoevaluación, de evaluación de iguales y de los docentes. La nota final se obtendría a través del proceso de gamificación.

\section{Aplicación del CIMA}

\section{Relato Resumido de las sesiones}

El primer día de la asignatura fue memorable. Tuvimos la suerte de que Julián Cosano, conserje de la facultad jubilado unos meses antes y muy querido por toda la facultad, se pasara por nuestra aula para animar al alumnado a participar en la donación de sangre que estaba teniendo lugar esos días. Después, Diego Jiménez y yo pasamos a explicar todo lo relacionado con la asignatura y la gamificación. Por último, durante los 20 minutos finales de la primera sesión de clase recibimos la visita de Ramón Blanco Barrera (@233art) docente de la Facultad de Bellas Artes que participa en el proyecto de innovación docente. Yo había visitado algunas de sus clases el cuatrimestre anterior y lo había invitado a mostrar a mi alumnado lo diferente que iba a ser esta asignatura este cuatrimestre. Ramón llevó a cabo una performance en la que empezó como Clark Kent y terminó como Superman encima de la mesa del profesor animando al alumnado a descubrir su talento y sus superpoderes y a volar muy alto con él. Lo más increíble fue que Diego, que no sabía nada de la visita a clase de Ramón ni de sus intenciones, también llevaba debajo de su ropa una camiseta de Superman. Así pues, empezamos la asignatura con tres superhéroes, Julián, Diego y Ramón, que iniciaron el cuatrimestre animando al alumnado, profesionales en formación, a desarrollar su talento y sus dotes creativas para dar el máximo de sí en la asignatura. 
El segundo día se llevó a cabo un cuestionario a través de la aplicación Mentimeter que complementaba otro de Google Forms que se había mandado al alumnado unas semanas antes del comienzo de las clases. En ambos cuestionarios se preguntaba al alumnado si estaba de acuerdo con las diferentes propuestas de los docentes con respecto a la asignatura acerca del modelo ABR con metodología DT/ECO, las actividades docentes que se iban a desarrollar en el aula y fuera del aula y cómo se iba a llevar a cabo la evaluación tanto formativa como sumativa. El alumnado mostró su apoyo casi unánime al uso de ABR con DT y a la gamificación tal como había sido diseñada por Diego. También mostró un apoyo mayoritario a la evaluación por medio de portafolios grupales en lugar de individuales, pero se decidió que fueran opcionales los ensayos que cada grupo tendría que proponer para que el resto de la clase pudiera profundizar en temas diferentes al de su propio reto. El único punto en el que el alumnado no mostró entusiasmo e incluso no hubo un apoyo mayoritario fue el de la participación en eventos como la Feria de la Ciencia, la MUAC, la Fiesta de la Historia, la Noche de los Investigadores, etc. Es por ello por lo que a diferencia del curso 2016/2017 en el que todos los grupos decidieron participar en la Feria de la Ciencia, este año se optó por no acudir a este evento y la participación del alumnado en los demás eventos se dejó como opcional, aunque incentivada a través de la gamificación. Solo se requeriría a todos los equipos presentar los resultados en el showroom final \#ECOshowUS para el que habrian de tener preparados también el póster y el vídeo de menos de menos de 5 minutos para contar lo vivido y conseguido en el proyecto en otros formatos y de una forma original y creativa.

La primera semana de clase se configuraron los grupos de trabajo en base a intereses comunes y con la única restricción apuntada anteriormente de la obligatoriedad de que hubiera españoles y extranjeros en los grupos. La lógica de esa restricción fue la experiencia de años 
anteriores en Lingüística Aplicada en los que la diversidad en los equipos había llevado a un trabajo más productivo y enriquecedor. Se comprobó que cuando se pone a trabajar juntos a Erasmus y españoles el ambiente de clase se transforma y que la complicidad que se crea aumenta la disposición para el buen trabajo en equipo.

Casi todas las sesiones de las clases presenciales se dedicaron al trabajo de los equipos en sus PIC. Cada equipo fue desarrollando las etapas del Design Thinking a su ritmo y según las necesidades de su reto. Los docentes nos dedicábamos a asesorar a los equipos y ayudarles a volver a seguir intentándolo cuando se equivocaban o se bloqueaban. También se invitó a antiguo alumnado a contar su experiencia con el trabajo en equipo, especialmente lo que había ido mal, y a otros docentes y a expertos para que pudieran dar asesoramiento. Vinieron también profesores participantes en el proyecto de innovación docente. El que más se implicó fue Eduardo Herrero que dedicó una clase completa a mostrar y hacer comprensible la metodología ECO. También dirigió en otra sesión la ejecución de la fase de prototipado pues yo me sentía inseguro con respecto a esta fase de la metodología y le pedí ayuda. Asistió asimismo a las presentaciones tanto intermedias como finales dando feedback al alumnado. Eduardo también cumplió la función de poner en cuestión todas y cada una de las ideas que el alumnado iba proponiendo con el fin de obligarles a ser más creativos.

Por último, el aprendizaje no se limitó al aula, sino que gracias a las redes sociales y a las actividades de divulgación en las que participamos se convirtió en ubicuo y permanente. Como canal alternativo y opcional de comunicación, usamos el grupo privado de Facebook LingSev que he venido empleando con éxito desde hace varios años en las dos asignaturas optativas de tercero y cuarto y anteriormente también en la asignatura de primero. Prácticamente la totalidad del alumnado matriculado se inscribió 
en el grupo y la inmensa mayoría participó activamente compartiendo los grandes hitos de la asignatura y muchos de los vídeos y fotos que se tomaban en clase, así como los productos que se iban generando y el conocimiento que se iba construyendo. La existencia de este grupo permitió que el alumnado que no podía asistir a clase regularmente pudiera participar en la asignatura e incluso llevar a cabo con éxito PIC individuales usando DT y ECO. Toda la información importante que se compartía en el grupo de Facebook era enviada también por email a todo el alumnado a través de la herramienta de anuncios de la plataforma de enseñanza virtual oficial de la US que sirvió además de repositorio para portafolios modelo y bastantes contenidos e información básica sobre la asignatura y la metodología usada. A mí personalmente el grupo privado de Facebook también me sirvió para saber más sobre el alumnado y aprenderme los nombres y apellidos de todos gracias a las continuas menciones. Aprenderme los nombres del alumnado es algo que no se me ha dado nunca bien en grupos grandes, pero que consigo desde que uso modelos docentes que dan mayor protagonismo al alumnado con la ayuda de las nuevas tecnologías.

\section{Evaluación del aprendizaje del alumnado.}

Con el modelo ABR con DT tal como se ha aplicado en Temas el alumnado aprende a trabajar en equipo e investigar sobre un tema, a empatizar, a detectar y abordar necesidades que tiene la sociedad en los que el lenguaje, la lingüística o la enseñanza de lenguas juegan un papel central, a hacer numerosas presentaciones en clase, a presentar la información en formato póster y formato vídeo, a defender sus ideas, a evaluar las ideas de los demás, a autoevaluarse y a reflexionar a través de un diario de clase. Aprende además a generar conocimiento por medio de la creación de una solución innovadora a un reto o necesidad de la sociedad. A través de su trabajo para encontrar 
e implementar esa solución y ayudar a los demás equipos a perfeccionar las suyas, expande sus conocimientos sobre diversos aspectos de la Lingüística y a aplicar esos conocimientos de forma creativa y eficaz. Aprende también a evaluar la solución a la que han llegado los demás equipos y la suya propia. Aprende a aceptar las críticas y a saber identificar si algo no ha ido bien para poder encontrar la raíz del problema y así aprender de los errores en el entorno seguro de la clase.

Lo más destacable fue que en lugar de una única historia, y una única voz, la del docente, en esta asignatura se crearon múltiples historias. Cada persona en la asignatura tuvo su propia voz como participante activo en la construcción global del conocimiento generado y compartido a través de los canales de comunicación, de los blogs y demás productos. Además, fueron protagonistas en eventos como la Fiesta de la Historia, las IV Jornadas de Sensibilización sobre la LSE en la UPO, la tercera edición de la MUAC, el Zoco de las Lenguas y Culturas el 24 de mayo de 2018 en la Facultad de Filología de la US y el \#ECOshowUS del 4 de junio. Las Jornadas de la UPO y el Zoco de las Lenguas y Culturas surgieron de las necesidades de dos de los proyectos del alumnado, el de enseñanza de español a refugiados y el de revitalización de la LSE.

Para evaluar formativamente se llevó a cabo una observación permanente de lo que iba haciendo cada equipo durante todo el cuatrimestre, hubo evaluación por pares de las presentaciones en clase, autoevaluación, evaluación anónima de los compañeros de equipo, evaluación de lo acontecido en clase y de los portafolios. El día del examen final, el 20 de junio, se estableció como fecha de entrega del portafolios grupal y se llevaron a cabo entrevistas de evaluación final del trabajo realizado y de la asignatura con cada uno de los equipos. Las notas obtenidas a través del proceso de gamificación en la convocatoria de junio tras las entrevistas y la evaluación de los portafolios 
fueron excelentes: diecinueve personas mantuvieron el 10 con el que comenzaron, dieciséis obtuvieron entre 9 y 10, 11 entre 8 y 9 , una persona obtuvo 7,7 y solo dos consiguieron una nota por debajo del notable pero cercana al 7. No obstante, se dio un plazo hasta el 14 de julio para que el alumnado pudiera escribir algunos de los ensayos propuestos por los otros equipos, o presentar un portafolios individual optativo para subir la nota final. El objetivo de este plazo extra de entrega de trabajos de la asignatura era que el alumnado pudiera profundizar en los proyectos de los demás equipos y poder determinar mejor quién recibiría las $\mathrm{MH}$ que se podían otorgar. Lamentablemente, el alumnado de último curso del Grado en Filología Hispánica está desbordado de trabajo en su último cuatrimestre con los TFG, las prácticas, las asignaturas pendientes de otros cursos, los trabajos remunerados y demás obligaciones con lo que este plazo para que profundizaran más en su aprendizaje no tuvo la repercusión que se hubiera deseado. Aún así, hubo varias personas que optaron por subir nota y por las $\mathrm{MH}$ con lo que los resultados finales de la asignatura fueron excelentes con $3 \mathrm{MH}, 34$ sobresalientes, 11 notables, 1 aprobado y 8 no presentados. Así pues, las 47 personas que asistieron regularmente a clase obtuvieron notas muy buenas en la convocatoria de junio. Además, dos de las tres personas que optaron por cursar la asignatura a distancia por no poder asistir a clase consiguieron finalizar sus PIC individuales con excelentes resultados. La tercera lo presentó en septiembre obteniendo una nueva $\mathrm{MH}$.

El proceso y los productos generados por el alumnado de Temas usando ABR con DT y gamificación fueron en general de mayor calidad que en otros cursos en los que se usaron otras metodologías docentes. Las presentaciones en clase también fueron mejores y sobre todo el alumnado mostró más originalidad y creatividad. La veracidad de estas afirmaciones se puede constatar si se examinan los productos generados (portafolios, vídeos-resumen, 
pósteres, blogs, etc.) y las grabaciones de vídeo de las presentaciones del curso 2016/2017 (Mora 2017b) y se comparan con las del 2017/2018.

\section{Evaluación del diseño puesto en práctica.}

El uso del ABR con DT/ECO y gamificación en la asignatura Temas durante el segundo cuatrimestre del curso 2017/2018 fue un éxito. El alumnado profundizó bastante más en sus temas y proyectos, si se compara con la experiencia del primer cuatrimestre en el que se usaron las mismas metodologías en la asignatura de tercero Lingüística Aplicada. Si la comparación se realiza con la misma asignatura Temas impartida el curso 2016/2017 con ABR pero sin DT ni gamificación, el contraste es mucho más notable, y aún mayor si se mira más atrás al primer cuatrimestre de 2016/2017 en el que en la asignatura Lingüística Aplicada se usó simplemente Aprendizaje Basado en Proyectos, con gran parte del alumnado sumándose a proyectos establecidos y creados por el docente. Muchos de los grupos de trabajo de las dos asignaturas en el curso 2016/2017 no llegaron a convertirse en verdaderos equipos por los problemas habituales del trabajo cooperativo y por la dificultad que conlleva el aprendizaje cooperativo cuando no se está acostumbrado a trabajar en equipo. Además, salvo excepciones, en el curso 2016/2017 no se profundizó tanto en los temas elegidos con lo que el aprendizaje fue más superficial. Con la metodología DT/ECO, que proporciona un modo de proceder para llegar a una solución creativa e innovadora al reto planteado, el alumnado llegó mucho más lejos. Por otro lado, la calidad y originalidad de los pósteres, los vídeos, los blogs (no obligatorios), los portafolios, las presentaciones parciales y finales creados por el alumnado fue superior, en general, tanto a los del primer cuatrimestre de Lingüística Aplicada como a los de cursos anteriores. Con respecto al primer cuatrimestre, los mejores resultados pueden deberse a que la inmensa mayoría 
del alumnado de Temas era de cuarto de grado mientras que la gran mayoría del alumnado de Lingüística Aplicada era de tercero, pero lo más probable es que la parte más sustancial de la gran mejora en los resultados se haya debido a que era la segunda ocasión en la que se implementaba DT/ECO. Es probable que también haya influido el no tener la distracción de los debates semanales ni de ensayos obligatorios, con lo que el estudiantado de Temas pudo dedicar más tiempo y centrarse más en profundizar en sus propios proyectos y retos.

Sin embargo, el mayor éxito de este CIMA fue que alumnado que no podía venir a clase pudo seguir la asignatura gracias al modelo docente personalizado empleado y sobre todo gracias al grupo de Facebook LingSev. Así, por ejemplo, la alumna María Cagigós llevó a cabo un gran PIC Viento del este, viento del Oeste en el que investigó cómo enseñar español de una manera más efectiva a inmigrantes chinos siguiendo DT y ECO plasmándolo en este gran blog: http://cagigos.blogspot.com/. Una segunda alumna que se encontraba en Lyon como auxiliar de conversación pudo también completar un PIC de gran calidad sobre enseñanza de español a alumnado francés de secundaria.

María Cagigós describió así en un correo electrónico enviado al docente su experiencia con el grupo LingSev de Facebook y la importancia del uso de nuevas tecnologías en clase:

El profesor Juan Pablo me ha pedido que hable de mi experiencia con las nuevas tecnologías. Recurre a mí por una razón sencilla y es debido a mi estado de salud, que me impide, entre otras muchas cosas, asistir a clase con regularidad. Ya había cursado en primero una asignatura de lingüística con él y sabía lo importante que es en sus clases la colaboración entre compañeros, la participación y el enriquecimiento mutuo. Por ese motivo, antes de matricularme y tenerlo de nuevo como profesor, quise escribirle y contarle cuál era mi situación. La respuesta fue un 
voto de confianza por su parte y puedo decir que he superado el reto con éxito y con muchísima satisfacción.

En mi caso particular ha sido especialmente importante el grupo de Facebook Lingsev. Me ha permitido estar totalmente conectada con mis compañeros y con lo que iban haciendo en el aula. Cuando uno no puede asistir a clase se encuentra realmente muy perdido, no sabes exactamente qué tienes que hacer, cómo llevarlo a cabo... y este grupo ha sido la herramienta que he utilizado para estar al día con los proyectos de mis compañeros, coger ideas, aportar sugerencias y recibir críticas y halagos muy constructivos, de una forma muy parecida a como lo habría hecho en clase. Es una plataforma muy motivadora porque genera debate, implica a los alumnos con temas que nos interesan, nos sorprenden y que, incluso, nos tocan la vena sensible, sacando lo mejor de nosotros porque la utilizamos de forma muy respetuosa. Son temas, además, que materialmente no podríamos tratar en el aula, por lo que aprendemos a convivir con la asignatura a lo largo de todo el día, también en nuestros ratos de ocio. Yo lo definiría como un proceso de encariñamiento con la materia.

Es cierto que la labor del profesor Mora es de total entrega con su alumnado, pero creo que, al final, el crecimiento es mutuo y todos nos beneficiamos de estas clases menos convencionales pero muy productivas.

Por esto creo que las nuevas tecnologías consiguen abordar la enseñanza desde otro ángulo con muy buenos resultados. Tal y como las usamos con Juan Pablo, no infantilizan de modo ninguno al alumno, ya que se nos exige para los trabajos, ensayos, etc. una parte de investigación, de contastación y documentación que llevamos a cabo con muchísima seriedad para que el resultado tenga nivel universitario. Es más, el enfoque práctico que le damos nos hace entrar muy de lleno en cada proyecto, sintiendo 
que lo que hacemos tiene una finalidad, aunque sea la de compartirla con el resto de alumnos. Esto, que parece una tontería, da sentido al esfuerzo, pues entendemos que no lo hacemos para nosotros, sino para los demás, por lo que debemos presentar algo que merezca la pena. Así desarrollamos una serie de aptitudes extremadamente importantes en nuestra vida, superando miedos, aprendiendo a defender y sacarle todo el jugo a un trabajo bien hecho, asumiendo errores, escuchando a los demás... Lo hacemos de forma amena, original, comunicativa...

En definitiva, creo que las nuevas tecnologías nos facilitan las cosas haciéndolas mejores, más provechosas.

El 20 de junio, durante las entrevistas finales se pasó un cuestionario anónimo de 55 preguntas elaborado en el marco del proyecto de innovación docente. Las cinco primeras preguntas trataban sobre la propuesta global de la innovación, las 13 siguientes sobre la planificación y desarrollo de la asignatura, las 5 siguientes sobre el estudiantado, las 8 siguientes sobre el profesorado, las 7 siguientes versaban sobre aprendizajes y orientación profesional y las 17 restantes sobre el proceso de Design Thinking. Aquí se puede acceder a las medias y gráficos de las respuestas de los 45 alumnos de Temas que completaron el cuestionario y que son en general bastante positivas:

https://hdvirtual.us.es/discovirt/index.php/s/ LjQeAmEsR9SdSod

A su vez, tras la entrega de las notas finales el 15 de julio se pasó un cuestionario a través de Google Forms muy parecido al que se pasó al alumnado de Temas el curso 2016/2017 (Mora 2017b). Se puede acceder a las respuestas a estos cuestionarios en este enlace: https://twitter.com/ jpmoragu/status/1021726714425225216 Se puede comprobar que la satisfacción del alumnado es muy notable, aunque por la fecha solo 27 respondieron. 
También se manifiesta la satisfacción del alumnado en los resultados de las encuestas oficiales de la Universidad de Sevilla que muestran la percepción que el alumnado tiene del docente, aunque fueran diseñadas y formulen preguntas pensadas para una docencia más bien tradicional. Voy a dar los datos para la asignatura Temas de 2016/2017, 2017/2018 y 2018/2019 de la media final de todas las preguntas y de la pregunta 18 (P18) que tiene como enunciado "En general, estoy satisfecho/a con la actuación docente desarrollada por este/a profesor/a". En el curso 2016/2017 la media fue de 4,11 sobre 5 y en la P18 se obtuvo un 4,03 con 37 encuestados. En el curso 2017/2018 la media fue de 4,14 y en la P18 se obtuvo un 4,03 con 35 encuestados. En el curso 2018/2019, la media fue de 4,25 y en la P18 se obtuvo un 4,50 con 43 encuestados. En la asignatura de primero fueron 4,35 y 4,46 con 33 encuestas (Mora 2019). Se ve la progresión y el avance a medida que se tiene más experiencia con ABR, aunque extraña que entre 2016/2017 y 2017/2018 no hubiera apenas diferencia.

No obstante, a diferencia de la experiencia del curso 2016/2017 en la que hubo varios grupos que no llegaron a convertirse en equipos y que tuvieron serios problemas que dificultaron que se llegara a buen puerto en los retos, en 2017/2018 solo hubo un grupo con serios problemas de comunicación entre el alumnado español y el Erasmus. A pesar de todo, se puede considerar que incluso de esa mala experiencia aprendieron, igual que sucedió en 2016/2017.

Me queda pendiente para los cursos siguientes lograr que haya un conocimiento más profundo del trabajo que están llevando los demás equipos de la asignatura. Además, veo necesario mejorar la estrategia de comunicación con el alumnado, ya que hubo quejas sobre los numerosos y largos mensajes que se enviaron por correo a través de la plataforma de enseñanza virtual. 
Por último, tengo que seguir evaluando el efecto de la gamificación. La gamificación es motivación fundamentalmente extrínseca y recuerda en todo momento la nota final. Yo soy partidario de intentar que el alumnado se olvide de la nota para intentar hacer crecer su motivación intrínseca. Siguiendo a Bain (2004), intento separar la evaluación formativa de la sumativa, que intento relegar al final del cuatrimestre. No obstante, tengo que reconocer que la gamificación funcionó muy bien y que la mayoría del alumnado mostró una gran satisfacción con ella, como se puede ver en el cuestionario final de Google Forms en el que 14 de los 27 estudiantes que lo respondieron dieron una calificación de 10 a la pregunta sobre el grado de satisfacción con la gamificación. Solo otra pregunta, la referida al grado de satisfacción con el portafolios, obtuvo un número tan elevado de dieces.

\section{Aspectos de la experiencia a incorporar en la práctica docente habitual}

Sin duda, voy a seguir usando el modelo de ABR con DT/ ECO pues me sirvió tanto para las asignaturas de las que he hablado aquí como para la dirección de uno de los TFM del máster MASELE que dirigi durante el curso 2017/2018. Mi propósito y nuevo CIMA para el curso 2018/2019 fue intentar probar el uso de este modelo docente también en la asignatura de primero Lingüística. Al ser una asignatura propedéutica, con un temario muy extenso y unos contenidos inabarcables en un cuatrimestre, no me había atrevido a ser tan disruptivo y el curso 2017/2018 seguí en primero el modelo de Flipped Learning complementado con Aprendizaje Cooperativo Basado en Proyectos que había venido usando desde el curso 2013/2014 y que me estaba funcionando cada vez mejor. La pregunta que me planteé fue si me funcionaría el ABR con DT/ECO también en primero. Ese es el gran reto que me propuse para el curso 2018/2019. Se puede leer en esta misma publicación 
sobre el éxito de este último CIMA que he llevado a cabo hasta la fecha (Mora 2019).

\section{Principios didácticos argumentados}

El curso académico 2017/2018 crecí enormemente como docente, mucho más que en todos los cursos anteriores. Fue gracias a la colaboración con el excelente equipo de profesionales del proyecto de innovación docente cuyas clases visité en varias ocasiones y que visitaron las mías. También fue gracias al apoyo de mis compañeras y compañeros de la REFID en cuyas cuartas jornadas de diciembre de 2017 tuve el privilegio de coordinar uno de los equipos de trabajo junto a Rosario Martínez y en las que una de mis alumnas, María José Maldonado, fue protagonista en la mesa redonda sobre la visión del alumnado acerca de la innovación docente. También colaboré con Jorge Ruiz y con Lola Limón, de la Facultad de Ciencias de la Educación, en un proyecto de creación de grupos inteligentes para la elaboración de los TFG y TFM que me enseñó mucho sobre cómo dirigir de forma más efectiva y enriquecedora Trabajos de Fin de Estudios, TFE (Escúchese el programa de Radius, TFEs en la Onda https://radio.us.es/programa/ tfes-en-la-onda/). Es sin duda a través de la colaboración con otros docentes como conseguimos dar lo mejor de nosotros y servir mejor a nuestro alumnado.

Voy a seguir en la senda de tener las más altas expectativas con respecto a mi alumnado. Casi nunca las defraudan y casi siempre las superan. Voy a seguir en la senda de ceder mayor control y responsabilidad al alumnado, de establecer una relación de confianza en la que se cuide el factor afectivo y se cree un buen ambiente de clase, para que cada alumna y alumno sienta que no son uno más, sino que todas y cada una son la persona más importante para mí en el aula y que se vean a sí mismas como futuras profesionales que tienen mucho que decir mientras 
aprenden y se forman. Voy a seguir también poniendo el foco en la sociedad y en sus necesidades pues estoy convencido de que el aprendizaje más efectivo se produce cuando se actúa sobre la realidad y el conocimiento y los contenidos que se generan de la materia se perciben como importantes para lograr cambios en la sociedad.

Todo ello con el fin de crear un entorno rico y natural de aprendizaje crítico en el que los futuros profesionales y docentes a los que tengo la suerte de enseñar se sientan seguros para probar nuevas soluciones que nos permitan aspirar a conseguir un mundo mejor.

Querría terminar este artículo reproduciendo las palabras que el 21 de julio de 2018 me envió una de las alumnas por correo electrónico, ya que son palabras como estas las que dan sentido a todo el esfuerzo y trabajo realizados tanto por mí como por el alumnado:

Acabo de completar la encuesta que nos has pasado y necesito escribirte estas palabras. No lo he hecho antes y lo hago ahora. Quería darte las gracias. Gracias por hacer que vuelva a confiar en la enseñanza de nuestro país. Gracias por entregarte de la forma en que lo haces con tus alumnos y con tu trabajo. Gracias por transmitirnos tu pasión por la lingüística. Quizá no hayas sido el profesor que más conocimientos técnicos de lingüística me ha dado pero, ¿Qué importa eso? Para mí desde luego no es lo más importante. Tú nos has enseñado otras muchas cosas. Personalmente, en mi proyecto me he superado a mi misma en muchos aspectos y he conseguido hacer cosas que jamás me habría imaginado que haría. Quizá no haya participado mucho en clase por mi condición o no haya ido al showroom (aunque me hubiera encantado) pero has conseguido, queriendo o sin querer, que crezca como persona en muchos sentidos. En primero ya me sorprendieron tus clases pero en cuarto te has superado con creces. Digo adiós a cuatro duros y, a la vez, maravillosos años. A ti 
te digo un hasta luego porque espero que sigamos en contacto por mucho tiempo más. Estos meses en tu asignatura, a pesar de los agobios y la saturación de trabajo que me invadía, me han dado el chute de energía y fuerza necesarias para lanzarme a todos los proyectos que me quedan por hacer en mi vida con el mismo entusiasmo que he hecho mi proyecto en grupo. De nuevo, gracias. Ahora me encuentro ante la vida real después de cuatro años en una especie de burbuja, pero lo hago con ganas, dispuesta a comerme el mundo, sin dejar que ningún miedo me coma, con madurez y fuerza para luchar por mis sueños. Siempre he tenido claro cuál sería mi profesión y aquí estoy, luchando por ella. Recuerda: nuestras armas son las ganas, la constancia, y la creatividad.

Todo un placer (y no me olvido de Diego. Estas palabras perfectamente podrian ir también para él). Yo para ti habré sido una alumna más pero te aseguro que tú no has sido un profesor cualquiera para mí. Gracias por devolverme la esperanza. Eternamente agradecida. 


\section{Referencias Bibliográficas}

Bain, K. (2004). What the Best College Teachers Do. Cambridge: MA. Harvard University Press. (Trad. Cast.: Lo que hacen los mejores profesores universitarios. Valencia, España: Publicaciones de la Universidad de Valencia).

Bergmann, J. y Sams, A. (2012). Flip your Classroom: Reach Every Student in Every Class Every day. Washington, DC: ISTE y Alexandria, VA: ASCD.

Bravo, E. (2016). Renovar la docencia Universitaria: reflexiones sobre la aplicación del ciclo de mejora en la asignatura Español de América. En R. Porlan y E. Navarro (Coords.), III Jornadas de Docencia Universitaria. SeviIla (págs. 431-444). Universidad de Sevilla-ICE.

Bravo-García, E. Ma (2017). Fomentar la independencia investigadora a través de la docencia. Una experiencia acerca de la docencia de "Español de América" en Máster. En R. Porlán y E. Navarro (Eds.). IV Jornadas de Formación e Innovación Docente. Sevilla: Universidad de Sevilla, 57-70.

Camacho-Taboada, V. (2019). Design Thinking en asignaturas propedéuticas de primero de Grado. Comunicación presentada a las VI Jornadas de Formación e Innovación Docente. Sevilla, España: Universidad de Sevilla.

Chou, Y. K. (2016). Actionable Gamification: beyond points, badges and leaderboards. Fremont, CA.: Octalysis Media.

Herrero, E. y Mora, J. P. (2018). Aprendizaje Basado en Retos con Design Thinking: ECO en el Grado en Filología Hispánica de la Universidad de Sevilla. Comunicación presentada al I Congreso Iberoamericano de Docentes, Universidad de Cádiz. Algeciras, del 6 al 8 de diciembre 2018.

Jiménez, D. (2017). ¿Qué es la gamificación? Boletín ASELE, $56,71-82$

Jiménez, D. (2018). Ciclo de mejora docente aplicado en Temas de Lingüística General. En Monográfico Jornadas de Formación e Innovación Docente del Profesorado. Vol. 1: 321-335.

Mancera, A. (2016). Enseñar Pragmática mediante la elaboración y el análisis de textos: una experiencia docente en el Grado en Filología Hispánica. En Porlán Ariza y Navarro Medina (Coords.) III Jornadas de Docencia Universitaria. Universidad de Sevilla (30 de junio y 1 de julio de 2016) (pp. 973-985). Sevilla, Espana: Universidad de Sevilla. 
Mcintosh, E. (2014). How to come up with great ideas and actually make them happen. Notosh Publishing. (Trad. cast.: Pensamiento de diseño en la escuela: Como lograr que surjan nuevas ideas y hacerlas realidad. Cádiz, España: SM, 2016).

Mora, J. P. (2015). Sóbre el mejor uso del tiempo de clase y las nuevas tecnologías para lograr un aprendizaje más significativo en grupos numerosos de Lingüistica, asignatura de primero de Filología. En R. Porlán y E. Navarro (Coords.), II Jornadas de Docencia Universitaria. Sevilla: Instituto de Ciencias de la Educación de la Universidad de Sevilla.

Mora, J.P. (2017a). Una experiencia de cambio docente en Fílología. En Porlán Ariza (Coord.) Enseñanza universitaria. Cómo mejorarla (págs.169-178). Madrid, España: Morata.

Mora, J.P. (2017b). Aprendizaje Basado en Retos o CBL (Challenge Based Learning) en la asignatura Temas de Lingüística General del Grado en Filología Hispánica. En R. Porlán y E. Navarro (Coords.), IV Jornadas de Formación e Innovación Docente (págs. 57-70). Sevilla, España: Universidad de Sevilla.

Mora, J. P. (2019). Comunicación presentada a las VI Jornadas de Formación e Innovación Docente. Sevilla, España: Universidad de Sevilla.

Porlán, R. (2017). Enseñanza Universitaria, Cómo mejorarla. Madrid, España: Editorial Morata.

Jornadas de Formación e Innovación Docente del Profesorado | № 2 (2019) Esta obra se distribuye con la licencia Creative Commons 